\title{
Multi-platform Tumour Profiling Delivers the Highest Clinical Utility and Improves Patient Outcomes in Today's Routine Clinical Practice
}

\author{
Giovanni Ussia $^{1^{*}}$, Robert Leonard ${ }^{2}$, Jaak Janssens ${ }^{3}$ \\ ${ }^{1}$ Dipartimento di Medicina e Chirurgia, OspedaleSant'Orsola, Universita di Bologna, Via Massarenti9, 40138 Bologna, Italia \\ ${ }^{2}$ Department of Surgery \& Cancer, Imperial College London, London, United Kingdom \\ ${ }^{3}$ Department of Oncology, Limburg Oncology Center, Belgium
}

\section{Abstract}

Empiric selection of drugs has a low rate of success in cancer patients whose treatment options are limited due to previous treatments or have rare cancer types with no standard effective treatment options. Guidance based on the molecular characteristics of the tumour can provide valuable insights for the physician as to which treatments could be beneficial and, conversely, which would be best to avoid. With the advent of rapid, affordable tumour-profiling technologies, many serviEces have been launched which offer patients and physicians this information. However, the field remains relatively unregulated and few services to date have demonstrated that their approach has meaningful clinical utility or clinical benefit with an acceptable cost-effectiveness ratio. In this review, we describe the concept and clinical data supporting the use of one such service, Caris Molecular Intelligence (CMI). Caris have been pioneers in the field of precision medicine for over a decade. Over 120,000 clinical publications describing the predictive nature of biomarkers were reviewed and graded to provide the basis for proprietary software that matches the published evidence with the test results from a multiplatform approach that measures proteins, ribonucleic acid (RNA) and deoxyribonucleic acid (DNA).The CMI results help physicians to select drugs that are more likely to benefit anindividual patient, avoid those drugs that are not likely to work, and provide treatment options that otherwise would not be considered. Globally, over 85,000 patients with over 150 different types of cancer have been profiled with CMI. The Caris approach has shown to be effective in 10 physician-led evaluations, and the results of a prospective observational registry show a significant overall survival benefit in patients treated in line with the report. In over 350 patients treated as part of a CMI evaluation, $75 \%$ have been treated based on the report and over $40 \%$ of those evaluable had clinical benefit. These outcomes help to illustrate the clinical utility of this approach in helping patients where the next treatment decision is difficult.

\section{Introduction}

Currently, standard of care (SOC) for patients with advanced cancer focuses on controlling the disease and its symptoms and extending the survival. In general, a 'one treatment fits all' approach is taken for SOC treatments, where all patients with a histologically defined cancer type are treated with a similar regimen or drug. While the overall response rate for cancer drug treatment has been estimated at 35\% [1], treatment for patients in whom SOC has failed is known to be less effective. A number of retrospective analyses have demonstrated diminishing response rates over successive lines of treatment in breast, colorectal and lung cancer [2-5].Among patients treated in phase I clinical studies, the overall response rate is reported to be around $6-10 \%[6,7]$.

Treatment of patients with advanced cancer is also associated with a high burden from drug toxicities, which is a particular problem if the drugs do not benefit the patients. These costs can be socially discriminating in healthcare systems where the patient has to pay for off-label treatment use.

The decision to continue treating with chemotherapy in advanced cancers is a complex process that requires oncologists to weigh up the risks and benefits. The patients may go on to receive therapies selected based on the physician's best knowledge, experience, recent literature of usually small studies, and clinical judgment("empirical" approach), or may receive best supportive care or may participate in clinical trials of new anticancer agents if they meet the typically strict eligibility criteria and have access to centres that can administer investigational agents.

\section{Publication History:}

Received: June 10, 2016

Accepted: August 29, 2016

Published: August 31, 2016

Keywords:

Multiplatform tumour profiling, Evidence-guided, Predictive biomarkers, Precision medicine, Oncology with a very difficult treatment decision, because for any patient, the likelihood of a benefit is extremely low and the financial burden high. Solving this therapeutic dilemma by finding the right drug for the right patient at the right time remains the goal of all personalised medicine approaches.

Advances in the discovery of prognostic and predictive biomarkers can provide oncologists with vital information which helps to stratify their patients for risk of tumour progression and identify potentially beneficial therapeutic agents based on biomarker expression patterns.

\section{Challenges in Demonstrating the Clinical Benefit of Precision Medicine in Oncology}

Evaluation of benefits from personalised medicine cannot rely on the traditional approach of demonstrating a benefit in a clinicallyselected patient group. Modern analytical tools have shed light on the

"Corresponding Author: Prof. Giovanni Ussia, Dipartimento di Medicina e Chirurgia, OspedaleSant'Orsola, Universita di Bologna, Via Massarenti9 , 40138 Bologna, Italia; E-mail: ussia.office@yahoo.co.uk

Citation: Ussia G, Leonard R, Janssens J (2016) Multi-platform Tumour Profiling Delivers the Highest Clinical Utility and Improves Patient Outcomes in Today's Routine Clinical Practice. Int J Surg Surgical Porced 1: 107. doi: http://dx.doi. org/10.15344/ijssp/2016/107

Copyright: (C) 2016 Ussia et al. This is an open-access article distributed under the terms of the Creative Commons Attribution License, which permits unrestricted use, distribution, and reproduction in any medium, provided the original author and source are credited. 
Citation: Ussia G, Leonard R, Janssens J (2016) Multi-platform Tumour Profiling Delivers the Highest Clinical Utility and Improves Patient Outcomes in Today's Routine Clinical Practice. Int J Surg Surgical Porced 1: 107. doi: http://dx.doi.org/10.15344/ijssp/2016/107

Page 2 of 8

enormous diversity between patients and no two patients are likely to share the same molecular alterations $[8,9]$. This diversity provides the basis for using personalised medicine and at the same time is the very reason for patients in comparative trials no longer having true controls.

Several uncontrolled studies have suggested clinical benefits from personalised therapy but the first randomised controlled trial to compare molecularly targeted treatments to conventional therapy, the SHIVA study, was negative. Several reasons may have contributed to the failure to demonstrate superiority of molecular profiling guided treatment selection in this study [10]. Firstly, the study focused on alterations in the PI3K pathway, the MAPK/ERK pathway and expression of hormone receptors. Biomarkers for the majority of cytotoxic chemotherapieswere not incorporated. Secondly, preclinical associations were used to guide clinical decision-making. For example, almost half of the patients received everolimus, based on an alteration in the PI3K pathway. Strong evidence demonstrated the effects of everolimus on a panel of 31 breast cancer cell lines [11]. Clinical data on the association of changes in the mTOR/PI3K pathway with everolimus activity are conflicting. While Hortobagyi et al. showed no association in ER+, HER- breast cancer patients, André and colleagues demonstrated a significantly prolonged progression free survival in hormone-receptor negative patients treated with everolimus [12,13] This example highlights the danger in extrapolating from what we observe in preclinical or animal models into what we expect to happen in patients. Experimental approaches with targeted therapies and decisions based on preclinical assumptions should be restricted to the confines of a clinical trial. It also highlights that approach to personalised medicine must be flexible to respond to emerging evidence, as our understanding of these complex pathways and interactions grows. The study used a fixed algorithm for selection of treatments. This takes away flexibility to take into account emerging knowledge and patient factors that need to be taken into account for the treatment decision, e.g. medical history, treatment history and individual physician preferences.

Many key academic centres have developed their own approach to precision medicine.The primary goal of precision medicine in most academic centres is to identify patients for enrolling clinical trials. Tsimberidou et al. published their experiences with tumour profiling at the MD Anderson Cancer Center. Over 1,100 patients were profiled to find 175 patients with a single aberration who could be treated using a matched treatment. While the exclusion of patients in whom no or many alterations could be found produced a high attrition rate, the outcomes of patients treated in line with profiling was exceptional. Patients enrolled in studies that required a matching genetic aberration had a response rate of $27 \%$, whereas only $5 \%$ of patients that could not be assigned to a trial based on molecular profiling responded to their selected treatment [14] In a similar approach at the Princess Margaret Cancer Center in Toronto, 643 patients were profiled to find 21 patients who received a therapy matched to a genetic aberration in their tumours [15]. Six of these 21 patients (29\%) had a confirmed partial response.

One major challenge is the number of patients that must be enrolled in these studies to find sufficient druggable alterations for selection of treatment. The majority of individual mutations are relatively rare, and the access to targeted therapies effective against these alterations outside clinical trials is difficult and often outside the financial reach of patients. As a result, the utility of 'Next Generation' sequencing alone is limited in routine clinical practice, with fewer than $20 \%$ of profiled patients receiving a treatment based on the results of sequencing [1619]]. Examining the proteins and RNA as well as the genetic sequence in a multiplatform approach greatly increases the number of possible targets and therefore possible treatment options.

\section{Evidence-Based, Multiplatform Approach to Tumour Profiling}

Over a decade ago, Dr. Daniel von Hoff pioneered the multiplatform approach that later became Caris Molecular Intelligence (CMI). His concept was to identify a clinically relevant and actionable molecular target for each of his individual patients. In order to enable this vision, biomarker results from across the central dogma of molecular biology - inclusive of DNA, RNA and proteins - would need to be examined using the appropriate technology platforms.

The approach taken has been constantly refined, resulting in a service, which is adapted to the latest scientific knowledge. To date, over 85,000 cases have been performed in over 150 tumour types, helping more than 5,000 ordering physicians in over 60 countries worldwide. CMI supports physicians in implementing actionable results from comprehensive tumour profiling in their routine practice. CMI is performed in a high-throughput laboratory that has been customized to accommodate large numbers of specimens for testing on multiple technology platforms. Comprehensive molecular testing is coupled with an evidence-based proprietary algorithm that translates complex biomarker results into two tables of drugs that are linked respectively with benefit or lack of benefit for that patient.

It is important that both laboratory developed tests (LDTs) and in vitro diagnostic tests (IVDs) are accurate so that health care providers do not seek unnecessary treatments, delay needed treatments, or expose patients to inappropriate therapies.

A panel of relevant, predictive biomarkers has been selected as the basis for the Caris system. These biomarkers are measured using an adequate methodology in relation to the clinical literature to maintain the predictive association (e.g. for a protein biomarker, it may not be relevant to measure gene expression). A number of validated high-throughput technology platforms are used, including immunohistochemistry, in situ hybridization, next generation sequencing, and pyro-sequencing. The panel of biomarkers and technologies used to measure them are continuously adapted and reflect emerging knowledge.

Proteins, gene expression, mutations and gene rearrangement can all have utility as predictive markers and better reflect the dynamic state of the tumour process. Taking an approach that is not reliant on a single technology is critical to perform clinically relevant biomarker testing. A pan-tumour approach to implementing predictive biomarker results is taken unless specific contradictory evidence is known.

The Caris approach involves continuous and extensive reviewing of therelevant and changing world literature and is overseen by senior expert scientists, pathologists and clinicians. An overview of the evidence process for CMI is shown in Figure 1.

Only predictive drug-biomarker associations that have been demonstrated in patients are considered for inclusion on the front page of the report - data from preclinical sources or animal models 
Citation: Ussia G, Leonard R, Janssens J (2016) Multi-platform Tumour Profiling Delivers the Highest Clinical Utility and Improves Patient Outcomes in Today's Routine Clinical Practice. Int J Surg Surgical Porced 1: 107. doi: http://dx.doi.org/10.15344/ijssp/2016/107

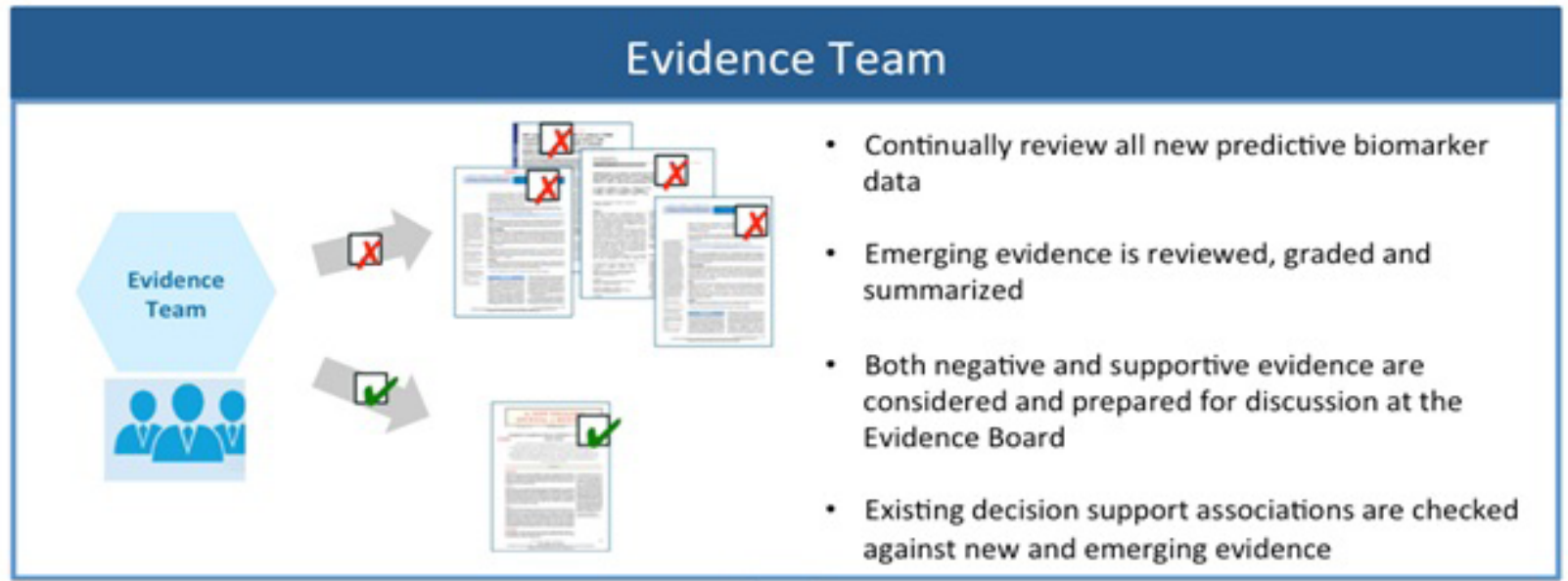

Graded and sent to

Evidence Board for consideration

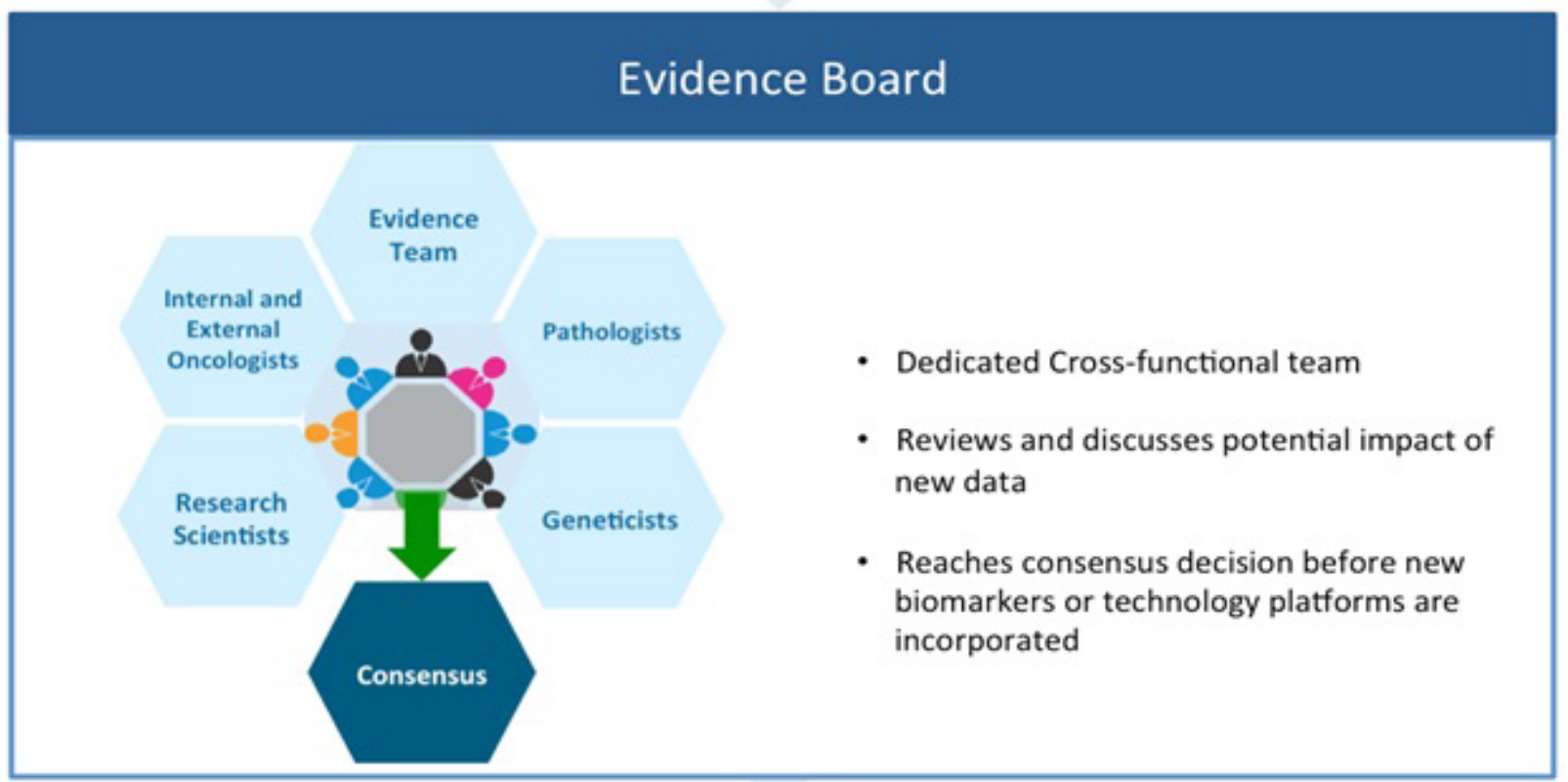

Evidence Board

approved

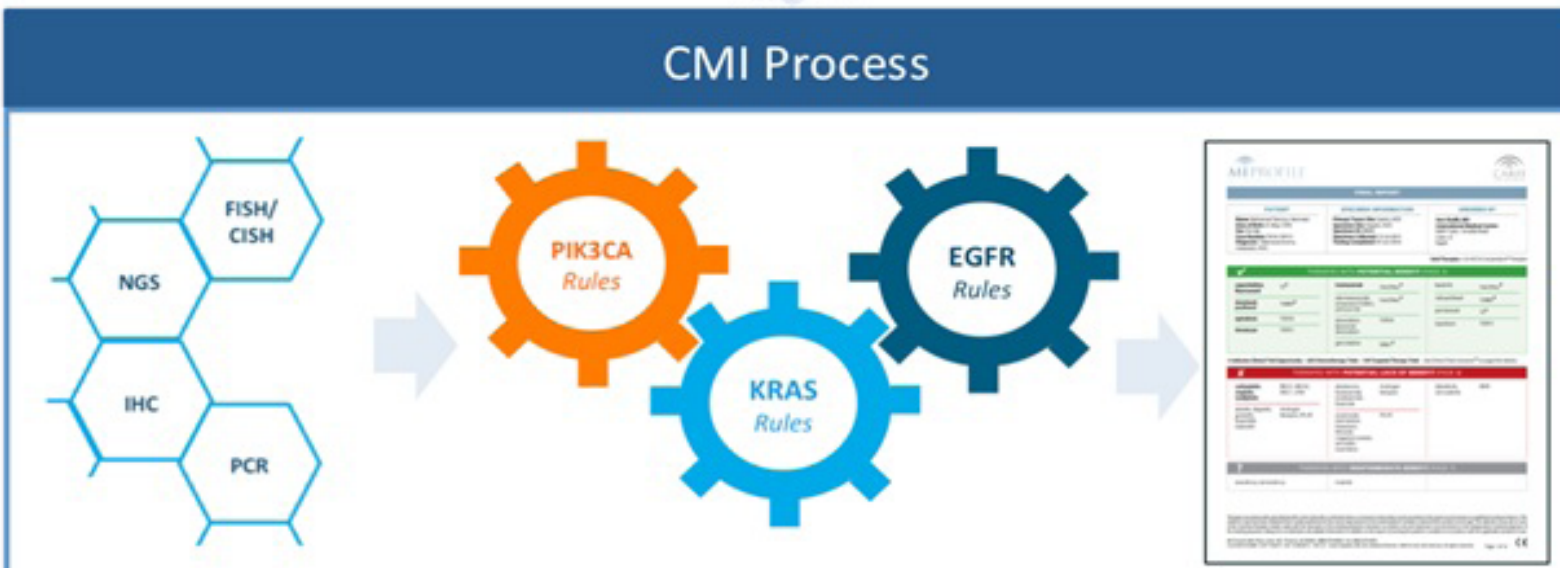

Figure 1: Generating Molecular Intelligence - An Overview of the Caris Evidence Process. 
is only taken into consideration for more experimental associations used to connect patients to enrolling clinical trials. Today, over $95 \%$ of drug/biomarker associations included in the service are supported by level 1 (randomised, controlled trials or meta-analyses) or level 2 (non-randomised, controlled trials, single arm or cohort/casecontrol analytic studies) evidence. Level 3 data includes examples such as the receptor tyrosine kinase (ROS1) fusion linked to crizotinib and activating HER2 mutations linked to trastuzumab in non-small cell lung cancer (NSCLC). Both of these biomarkers have been included in the National Comprehensive Cancer Network (NCCN) guidelines on the basis of level 3 evidence. The service includes clinical associations for over 60 drugs that are based on a multiplatformtumour profile of more than 80 biomarkers.

The report results are provided to the treating physician and supported by the relevant references from the peer-reviewed literature as determined bythe evidence review process. The report also enables the treating physician to review in detail the biomarker testing that has been performed, as well as link directly to the clinical evidence supporting the biomarker-drug association. Caris offers to explain every report in detail to the treating physician by telephone or a written consultation.

\section{Clinical Evidence Supporting the Use of CMI}

The pilot study of using molecular profiling of patients' tumours to find potential targets and select treatments for their refractory cancers, also known as the Bisgrove study, was conducted by Dr DanielVon Hoff and colleagues towards the end of the last decade [20]. Their innovative study is regarded as a cornerstone in the field of oncology precision medicine and has been cited over 70 times. The assumption made by the authors was that the molecular profile of each patient's cancer would be unique and that this would be more relevant to the treatment decision than the tumour type that was diagnosed. For this reason, individual patients were used as their own control, and the progression-free survival (PFS) on molecular-guided treatment compared to the PFS on the prior line of treatment. If the observed PFS ratio was greater than 1.3 (indicating a 30\% increase in PFS with profiling-guided treatment), the patient was considered to have clinical benefit.

Nine other physician-led evaluations of CMI have been conducted globally in a variety of solid tumours [21-28]. The definition of clinical benefit differed between studies and includedPFS ratio as described by von Hoff to more traditional endpoints such as disease control rate, response or overall survival greater than 6 months. To date, 486 patients have been included in these evaluations and 364 (75\%) have been treated in line with the findings of the report. The lowest utility was observed in the studies enrolling refractory pancreatic and gastric cancers, where the aggressive nature of the disease led to patients progressing before they could receive CMI-guided treatment.A prospective study of CMI use in Austria was still enrolling at the time of the first snapshot so not all patients had been treated yet [27].

A study from the Caris registry including 1180 patients with various solid tumours found a 422 days extension in the median overall survival of patients treated in line with the report compared to those who received at least one unmatched therapy [30]. A recent study has shown that electronic medical record data from over 4,700 patients showed a modest but significant increase in the time to next therapy, a surrogate for PFS, of 33 days in those patients who received a treatment predicted to be of benefit according to the CMI report [31].

\section{Conclusion}

It is recognised that in the clinic, human cancers usually comprise diverse populations of varying genotype, phenotype and hence probable variable sensitivity to anti-cancer therapy. This heterogeneityis rarely addressed in the planning of treatment beyond the frequent use of combinations of individually active drugs when the patient is first treated. Relapse of cancer means that the initially selected drugs failed to deal adequately with this heterogeneity. Furthermore, the molecular state of the relapsed cancer is highly likely to have changed compared to that of the initial state. It is therefore not surprising that the success of empirically chosen treatments for first and second relapses is never satisfactory and rapidly diminishes over the natural history of the disease. For this reason alone, curing relapsed metastatic cancer is not routinely a realistic goal of treatment. However, clinical response, as judged by good palliation of symptoms accompanied by some evidence of cancer regression, can be a realistic objective of treatment. The challenge is how to use our collective scientific knowledge to maximize this possibility for individual patients whose cancers, as have been revealed by study of their nucleic acids and proteins, are biologically unique to them.

There is a clear unmet medical need for cancer patients who are past standard of care therapy options but still fit and willing to receive further treatment. Although guidelines recommend that these patients enter either clinical trials or palliative care, suitable trials may not be available though the patients are often fit and willing to receive further anti-cancer treatment. Many of these patients are considered difficult to treat by the physician, as there is no clear guidance on the next, most effective treatment to use. The advent of precision medicine has brought hope that leveraging our growing knowledge of molecular alterations in cancers can lead to better treatment selection. Comprehensive tumour profiling can identify active treatments, help avoiding treatment which are likely not active, and find treatments that would not otherwise be considered.

Comprehensive tumour profiling gives a detailed snapshot of the tumour at a fixed time-point in the tumour's evolution. It is widely acknowledged that there can be heterogeneity between the primary and metastatic lesions and that the biomarkers are in a dynamic state, with changes in biomarkers reflecting selective pressures from chemotherapies or changes in the tumour microenvironment. Protein changes, alterations in RNA and even accumulation of further mutations can occur in the same tumour over time. For this reason, it is recommended that tumour profiling should be performed on a recent biopsy where possible and if deemed safe to do so in the individual patient. This is likeliest to give the most accurate assessment of the best treatment options for the patient at that time to help select targeted therapies where alterations associated with sensitivity or resistance can occur.

To date, many narrow approaches have failed to deliver sufficient clinical utility to become established in the clinical guidelines. The reason for the limited clinical utility today is lack of actionable alterations that can be effectively treated using available targeted therapies or clinical trials. Gaining access to investigational agents that act against many of the alterations that can be foundrepresents a difficult challenge due to a paucity of appropriate clinical trials. This can dramatically limit the clinical utility of services focused on 'Next Generation' sequencing alone. However, the recent evaluations of a comprehensive multiplatform approach in routine clinical practice 
Citation: Ussia G, Leonard R, Janssens J (2016) Multi-platform Tumour Profiling Delivers the Highest Clinical Utility and Improves Patient Outcomes in Today's Routine Clinical Practice. Int J Surg Surgical Porced 1: 107. doi: http://dx.doi.org/10.15344/ijssp/2016/107

a) Refractory solid Cancers - PFS Ratio (Von Hoff et al.)

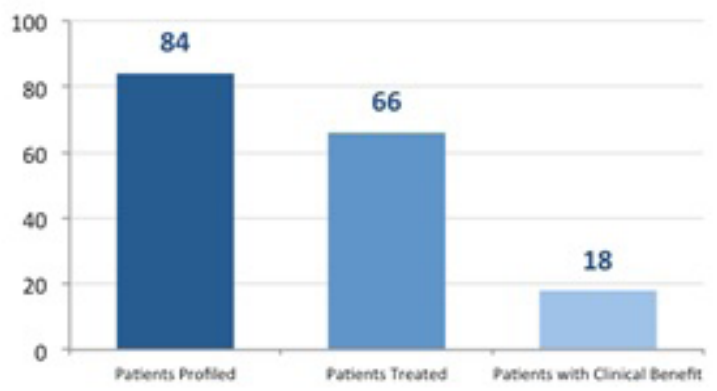

c) Refractory Breast Cancers - PFS Ratio (Jameson et al.)
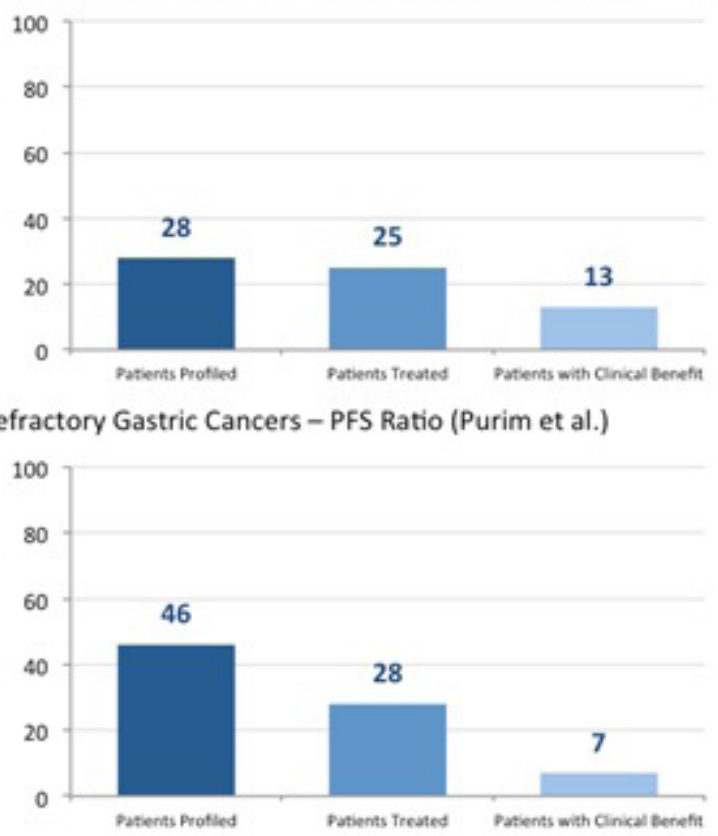

e) Refractory Gastric Cancers - PFS Ratio (Purim et al.)

b) Refractory Pancreatic Cancers - PFS Ratio (Epelbaum et al.)

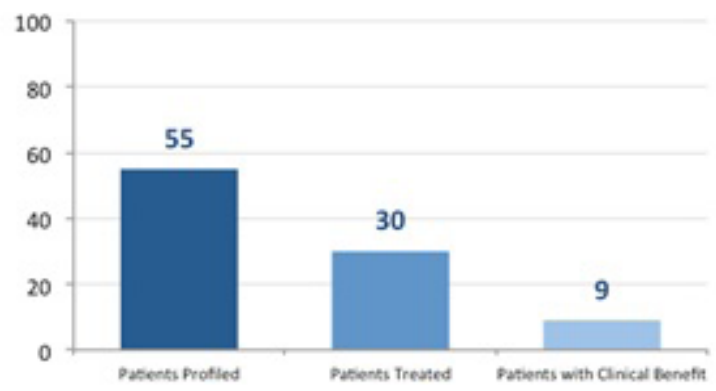

d) Refractory Solid Cancers - Disease Control (Dean et al.)

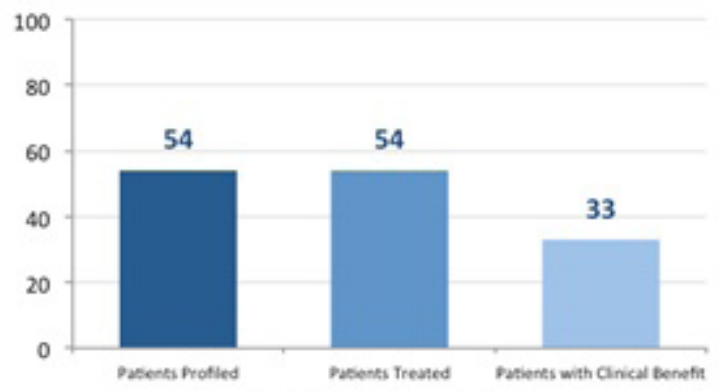

f) Metastatic ACCs - CR/PR/SD>6 months (Popovtzer et al.)

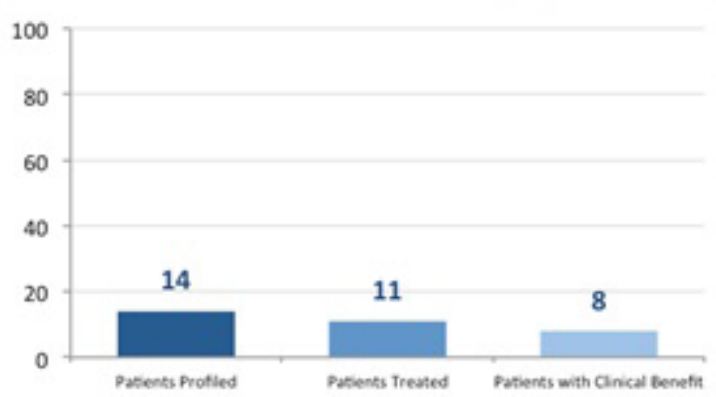

g) Refractory Solid Cancers - RECIST Response (EI Ahmadie et al.) h) Refractory Solid Cancers - PFS Ratio (Seeber et al.)

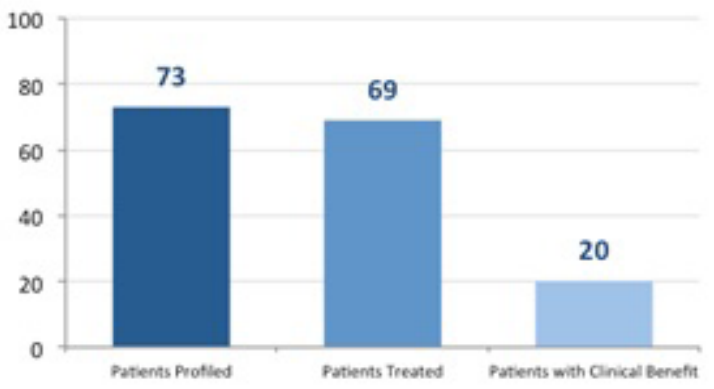

i) Refractory Solid Cancers - PFS Ratio (Chahine et al.)

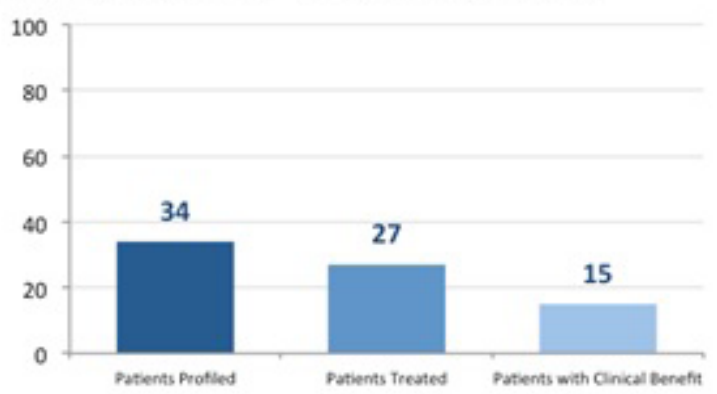

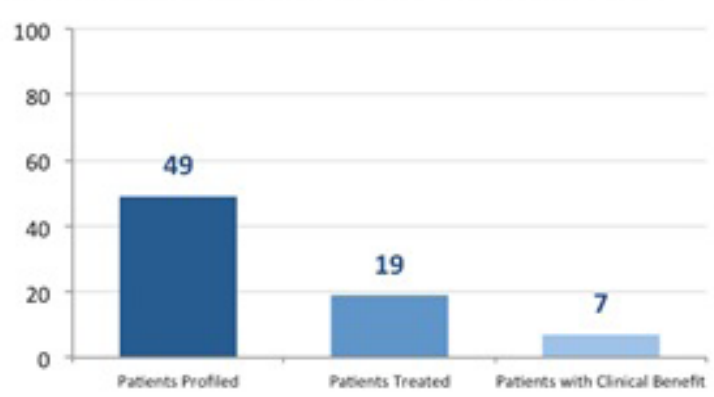

j) Refractory Pancreatic Cancers - OS>6 months (Ramanathan et al.)

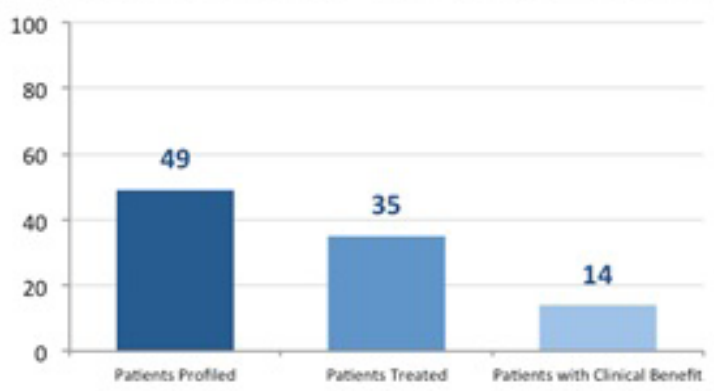

Figure 2: An overview of the ten physician-led studies of CMI showing observed rates of clinical utility and clinical benefit. 
Citation: Ussia G, Leonard R, Janssens J (2016) Multi-platform Tumour Profiling Delivers the Highest Clinical Utility and Improves Patient Outcomes in Today's Routine Clinical Practice. Int J Surg Surgical Porced 1: 107. doi: http://dx.doi.org/10.15344/ijssp/2016/107

\section{Matched}

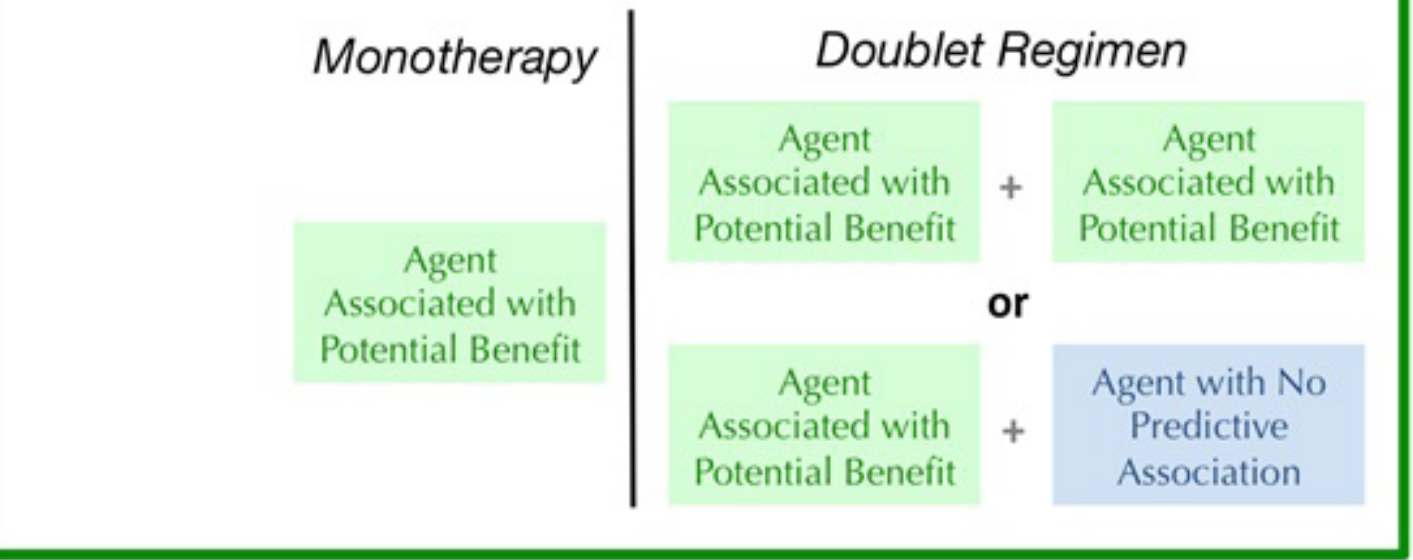

\section{Unmatched}

\section{Monotherapy}

Agent Associated with Lack of Potential Benefit

\section{Doublet Regimen}

Agent

Associated with

Potential Benefit

or

Agent Associated

with Lack of

Potential Benefit

or

Agent Associated

with Lack of

Potential Benefit
Agent Associated with Lack of

Potential Benefit

Agent with No Predictive Association

Agent Associated with Lack of Potential Benefit

\section{Excluded}

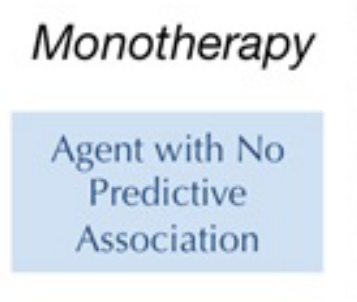

\section{Doublet Regimen}

Agent with No

Predictive

Association
Agent with No

$+$

Predictive

Association

Figure 3: Cohort definition for demonstration of clinical utility using registry databases or electronic medical records. Treatments are classed as matched, unmatched or excluded for the purposes of analysis. 
Citation: Ussia G, Leonard R, Janssens J (2016) Multi-platform Tumour Profiling Delivers the Highest Clinical Utility and Improves Patient Outcomes in Today's Routine Clinical Practice. Int J Surg Surgical Porced 1: 107. doi: http://dx.doi.org/10.15344/ijssp/2016/107

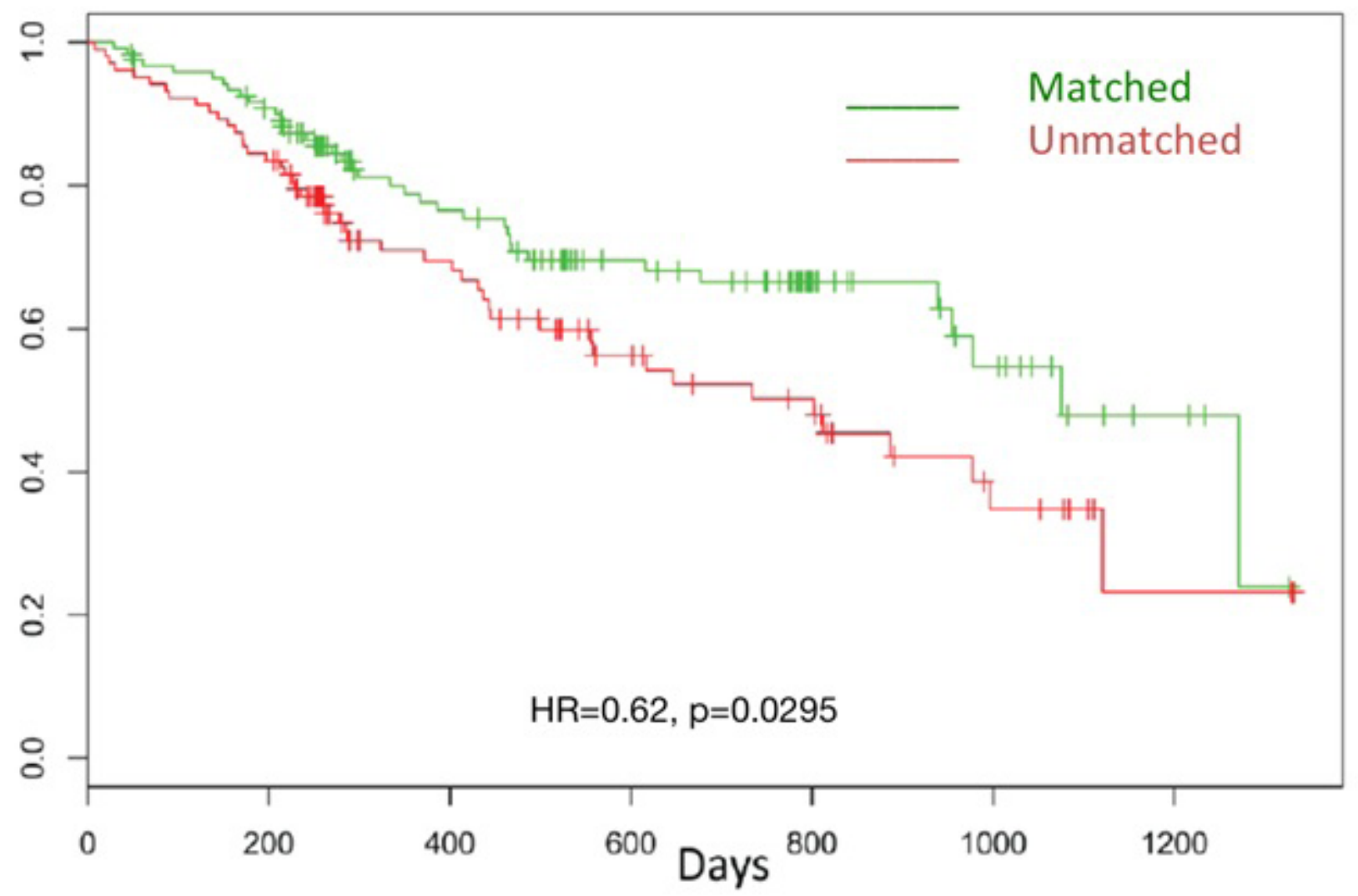

Figure 4: Kaplan-Meier curve showing the increase in overall survival from time of profiling for those patients treated only with therapies predicted to be of benefit by their molecular profile compared to those patients who received at least one therapy predicted to be lack of benefit [29].

have used novel and innovative methods to showpromising levels of clinical utility. The majority of patients profiled are treated in line with the report and a significantly longer overall survival has been observed in many patients. Further evaluation of this method in wider real-world scenarios is warranted. In March 2016,in the United Kingdom, the NHS began an evaluation of CMI in patients with refractory or rare ovarian and gynecological cancers. The promising results observed with CMI prove that the unique approach Caris Life Sciences has taken - comprehensive, continuous study of the medical literature, highest quality multi-technology platform laboratory assessments and an actionable report that gets reviewed and explained to the treating physicians by an expert in the field - is addressing the medical need in patients with advanced cancers. With its "easy to read" report, expert help and high clinical utility, this service is readily applicable in each cancer center, whether university, regional hospital and private practice.

\section{Competing Interests}

The authors declare that they have no competing interests.

\section{References}

1. Jackson DB (2009) Clinical and economic impact of the non-responder phenomenon - implications for systems based discovery. Drug Discov Today 14: 380-385.

2. Planchat E, Abrial C, Thivat E, Mouret-Reynier MA, Kwiatkowski F, et al. (2011) Late lines of treatment benefit survival in metastatic breast cancer in current practice? Breast 20: 574-578.
3. Bailey CH, Jameson G, Sima C, Fleck S, White E, et al. (2012) Progressionfree Survival Decreases with Each Subsequent Therapy in Patients Presenting for Phase I Clinical Trials. J Cancer 3: 7-13.

4. Palumbo R, Sottotetti F, Riccardi A, Teragni C, Pozzi E, et al. (2013) Which patients with metastatic breast cancer benefit from subsequent lines of treatment? An update for clinicians. Ther Adv Med Oncol 5: 334-350.

5. Reinmuth N, Payer N, Muley T, Hoffmann H, Herth FJ, et al. (2013) Treatment and outcome of patients with metastatic NSCLC: a retrospective institution analysis of 493 patients. Respir Res 14: 139

6. Von Hoff DD, Turner J (1991) Response rates, duration of response, and dose response effects in phase I studies of antineoplastics. Invest New Drugs 9: 115-122.

7. Olmos D, A'hern RP, Marsoni S, Morales R, Gomez-Roca C, et al. (2012) Patient selection for oncology phase I trials: a multi-institutional study of prognostic factors. J Clin Oncol 30: 996-1004.

8. Schwaederle M, Parker BA, Schwab RB, Fanta PT, Boles SG, et al. (2014) Molecular tumor board: the University of California-San Diego Moores Cancer Center experience. Oncologist 19: 631-636.

9. Egas-Bejar D, Anderson PM, Agarwal R, Corrales-Medina F, Devarajan E et al. (2014) Theranostic Profiling for Actionable Aberrations in Advanced High Risk Osteosarcoma with Aggressive Biology Reveals High Molecular Diversity: The Human Fingerprint Hypothesis. Oncoscience 1: 167-179.

10. Le Tourneau C, Delord JP, Gonclaves A, Gavoille C, Dubot C, et al. (2015) Molecularly targeted therapy based on tumour molecular profiling versus conventional therapy for advanced cancer (SHIVA): a multicentre, openlabel, proof-of-concept, randomised, controlled phase 2 trial. Lancet Oncol 16: $1324-1334$

11. Weigelt B, Warne PH, Downward J (2011) PIK3CA mutation, but not PTEN loss of function, determines the sensitivity of breast cancer cells to mTOR inhibitory drugs. Oncogene 30: 3222-3233. 
Citation: Ussia G, Leonard R, Janssens J (2016) Multi-platform Tumour Profiling Delivers the Highest Clinical Utility and Improves Patient Outcomes in Today's Routine Clinical Practice. Int J Surg Surgical Porced 1: 107. doi: http://dx.doi.org/10.15344/ijssp/2016/107

12. Hortobagyi GN, Chen D, Piccart M, Rugo HS, Burris HA 3rd, et al. (2016) Correlative Analysis of Genetic Alterations and Everolimus Benefit in Hormone Receptor-Positive, Human Epidermal Growth Factor Receptor 2-Negative Advanced Breast Cancer: Results From BOLERO-2. J Clin Oncol 34: 419-426.

13. André F, Hurvitz S, Fasolo A, Tseng LM, Jerusalem G, et al. (2016) Molecular Alterations and Everolimus Efficacy in Human Epidermal Growth Factor Receptor 2-Overexpressing Metastatic Breast Cancers: Combined Exploratory Biomarker Analysis From BOLERO-1 and BOLERO-3. J Clin Oncol 34: 2115-2124

14. Tsimberidou AM, Iskander NG, Hong DS, Wheler JJ, Falchook GS, et al. (2012) Personalized medicine in a phase I clinical trials program: the MD Anderson Cancer Center initiative. Clin Cancer Res 18: 6373-6383.

15. Bedard PL, Ozo AM, Tsao MS, Leighl NB, Shepherd FA, et al. (2013) Princess Margaret Cancer Centre integrated molecular profiling in advanced cancers trial (IMPACT) using genotyping and targeted nextgeneration sequencing. J Clin Oncol 31, (suppl; abstr 11002)

16. Ferreira AR, Gomes ASDA, Costa FP, Saragiotto DF, Katz A, et al. (2014) Next generation sequencing - A key in search of locks? Ann Oncol 25 (suppl 4): iv560.

17. Sohal DP, Rini BI, Khorana AA, Dreicer R, Abraham J, et al. (2015) Prospective Clinical Study of Precision Oncology in Solid Tumors. J Nat Cancer Inst 108.

18. Powell SF, Dib EG, Bleeker JS, Keppen MD, Mazurczak M, et al (2016) Optimizing Genotype Matched Clinical Trail (GMCT) accrual in a community oncology program (GOP). J ClinOncol 34 (suppl; abstr e18036).

19. Gupta D, Zook S, Kuhah H, Nazari A, Kramer K, Crilley PA, et al. (2016) Key drivers of physician decision-making in utilizing next generation sequencing results to guide cancer therapy. J ClinOncol 34 (supplabstr e18101)

20. Von Hoff DD, Stephenson JJ Jr, Rosen P, Loesch DM, Borad MJ, et al (2010) Pilot study using molecular profiling of patients' tumours to find potential targets and select treatments for their refractory cancers. J Clin Oncol 20: 4877-4883

21. Jameson GS, Petricoin EF, Sachdev J, Liotta LA, Loesch DM, et al. (2014) A pilot study utilizing multi-omic molecular profiling to find potential targets and select individualized treatments for patients with previously treated metastatic breast cancer. Breast Cancer Res Treat 147: 579-588.

22. Dean A, Byrne A, Marinova M, Hayden I (2016) Clinical outcomes of patients with rare and heavily pretreated solid tumours treated according to the results of tumour molecular profiling. BioMed Res Int 2016: 4627214

23. Popovtzer A, Sarfaty M, Limon D, Marshack G, Perlow E, et al. (2015) Metastatic Salivary Gland Tumours: A Single-center Study Demonstrating the Feasibility and Potential Clinical Benefit of Molecular-profiling-guided Therapy. BioMed Res Int 2015: 614845.

24. Ramanathan RK, Barrett M, Weiss GJ, Posner R, Rajeshkumar NV, et al (2012) Abstract nr LB-221: Phase II study of Therapy Selected by Tumour Profiling in Patients with Previously Treated Metastatic Pancreatic Cancer. A Study of the SU2C Consortium. Cancer Res 72: Supplement.

25. Epelbaum R, Shacham-Shmueli E, Klein B, Agbarya A, Brenner B, et al (2015) Molecular Profiling-Selected Therapy for Treatment of Advanced Pancreaticobiliary Cancer: A Retrospective Multicenter Study. BioMed Res Int 2015: 681653

26. Purim O, Shacham-Shmueli E, BenyAet al. (2015) Molecular profiling selected treatment in metastatic gastric and esophageal cancer: Real-life clinical experience. BioMed Res Int

27. Seeber A, Gastl G, Ensinger C et al. (2016) Treatment of Patients with Refractory metastatic Cancer according to Molecular Profiling on Tumour Tissue: An Interim-Analysis of the ONCO-T-PROFILE Project. Oncotarget (In Press)

28. Chahine G, Nasr F, Saleh K et al. (2016) Clinical outcomes of patients with previously treated advanced or metastatic cancer before or after tumour profiling: results of a monocentric pilot study in Lebanon. Presented at ICACT 2016

29. Herzog TJ, Spetzler D, Xiao N, Burnett K, Maney T, et al. (2016) Impact of molecular profiling on overall survival of patients with advanced ovarian cancer. Oncotarget 7: 19840-19849.
30. Spetzler D, Xiao N, Burnett K, Burch K, Abbott B, et al. (2015) Multi-platform molecular profiling of 1,180 patients increase median overall survival and influences treatment decision in $53 \%$ of cases. European Journal of Cancer 51(Supplement S3): S44

31. Marshall J, Schwartzberg LS, Bepler G, Spetzler D, El-Deiry WS, et al. 2013) Novel panomic validation of time to next treatment (TNT) as an effective surrogate outcome measure in 4,729 patients. J Clin Oncol 34(suppl; abstr 11521) 